\title{
Melanoma melanocítico oral em cão - revisão de literatura
}

\section{Hellen Magela Barreto}

Discente do curso de Medicina Veterinária UBM (Barra Mansa, RJ, Brasil) hmagelabarreto@gmail.com.

\section{Marcus André Ferreira Sá}

Doutorando em Medicina Veterinária pela Universidade Federal Rural do Rio de Janeiro - UFRRJ e professor de Reprodução Animal e Obstetrícia Veterinária no curso de Medicina Veterinária do Centro Universitário de Barra Mansa - UBM (Barra Mansa, RJ, Brasil)

marcus.ferreira85@hotmail.com 


\section{Resumo}

O melanoma é descrito como uma desordem neoplásica proveniente da transformação maligna do melanócito normal, que é responsável pela produção de melanina. Acomete principalmente a cavidade oral, junções mucocutâneas e pele de cães. Apresenta-se como um tipo de câncer com pior prognóstico e com alta incidência de metástase. Este trabalho tem por objetivo apresentar uma revisão da literatura, abordando a etiologia e fisiopatologia do melanoma oral, terapêutica, incluindo procedimentos cirúrgicos, quimioterápicos, radioterápicos, imunoterápicos e eletroquimioterápicos.

Palavras-chave: Melanócitos. Oncologia. Cirurgia. Cães.

\section{Abstract}

Melanoma is described as a neoplastic disorder originating from malignant transformation of the normal melanocyte, which is responsible for the production of melanin. It primarily affects the oral cavity, mucocutaneous junctions and the skin of dogs. It is presented as one of the cancer types with worse prognosis and with a high incidence of metastasis. This work aims to present a literature review, addressing the etiology and pathophysiology of oral melanoma, therapeutic, including surgical, chemotherapeutic, radiotherapeutic, immunotherapeutic and electrochemotherapeutic procedures.

Keywords: Melanocytes. Oncology. Surgery. Dogs. 


\section{Introdução}

Em virtude da elevada frequência de atendimentos oncológicos realizados na rotina veterinária, essa especialidade se tornou uma das principais no âmbito da Medicina Veterinária (MACEWEEN et al., 2001).

De acordo com Zamboni et al. (2011), o melanoma pode ser conceituado como uma desordem neoplásica proveniente da transformação maligna do melanócito normal, responsável pela produção de melanina. Os melanomas representam 7\% de todas as neoplasias malignas que atingem os cães, acometendo principalmente cavidade oral, região sublingual e junções mucocutâneas. Apresenta-se como um tipo de câncer com pior prognóstico e alta incidência de metástase. Cerca de apenas 5\% dos animais portadores sobrevivem por um período de cinco anos (SMITH et al., 2002).

Baseando-se no último boletim da OMS (1998), os melanomas são classificados, quanto ao seu aspecto morfológico, em três subtipos histológicos: epitelioide, fusiforme e misto. E quanto ao seu fenótipo referente à síntese de melanina pode ser classificado em melanóticos e amelanóticos.

Os estudos sobre neoplasias em animais objetivam descobrir métodos diagnósticos mais precisos, novos tratamentos que favoreçam o prognóstico da enfermidade, além de servir como base para estudos em humanos, a fim de se compreender o comportamento dos melanomas em ambas as espécies.

\section{Revisão de Literatura}

\section{ETIOLOGIA}

Vários fatores etiológicos estão envolvidos nos melanomas malignos em cães, como: consanguinidade, trauma, exposição química, hormônios e susceptibilidade genética (SMITH et al., 2002). No entanto, não há consenso quanto à etiologia dos melanomas malignos caninos. Em humanos, os principais fatores de risco incluem histórico familiar, características da pigmentação da mucosa e exposição ao sol, particularmente aos raios UVB (HAASS et al., 2010). 
Entretanto, a incidência de luz solar provavelmente não está envolvida no desenvolvimento de melanomas de mucosa, como os da cavidade oral, em cães. Outros fatores- como a presença de células pigmentadas ou mesmo a microbiota bucal e a inflamação- podem estar associados à etiologia desses tumores (DZUTSEV et al., 2015).

Embora os melanomas possam surgir em qualquer cão, a prevalência dessa doença é maior em animais de raça pura, especialmente Schnauzers padrão e miniatura, Pinschers, Doberman, Scottish Terriers, Irlandês, Gordon Setters e Golden Retrievers. Esse fato demonstra que os melanomas em cães apresentam influência genética (MODIANO et al., 1999). Modificações genéticas e epigenéticas nos melanócitos que levam a alterações na expressão ou função de genes e proteínas envolvidos no controle do ciclo celular e apoptose estão certamente envolvidos no desenvolvimento de melanomas (LIPTAK et al., 2013).

De acordo com Ramos-Vara et al. (2000), os cães acometidos por melanoma oral apresentam idade média de 11,4 anos, sendo que os machos estão mais predispostos do que as fêmeas a desenvolverem melanomas orais malignos.

\section{EPIDEMIOLOGIA}

Os dados obtidos a partir de estudos históricos indicaram que as seguintes raças de cães são consideradas mais suscetiveis a desenvolver tumores melanocíticos: Airedale Terrier, Boston Terrier, Boxer, Chihuahua, Chow Chow, Cocker spaniel, Pinscher, Doberman, Spaniel de Springer inglês, Golden Retriever, Setter Irlandês (PRIESTER, 1973). Entretanto, mais recentemente, raças como Schnauzer Miniatura, Scottish Terrier, Poodles, RottweillersREVER e Labrador Retrievers também foram descritas (GILLARD et al., 2014).

Segundo Tedardi et al. (2014), os registros dos animais acometidos por neoplasias são as fontes mais importantes de dados epidemiológicos para o assunto. Porém, existem apenas alguns sistemas de registro de animais em todo o mundo, sendo que os mesmos estão situados principalmente na Europa e nos EUA. Diante disso, em 2013, criou-se o Registro de Câncer de Animais de São Paulo na Universidade de São Paulo (USP), sendo o primeiro na América Latina (TEDARDI et al., 2014; 2015). Este 
sistema permite o desenvolvimento de estudos epidemiológicos mais precisos em animais domésticos dessa região.

Em estudo retrospectivo, realizado entre os anos de 1993 a 2002, envolvendo 1813 casos de neoplasias em cães, 58 apresentavam neoplasias melanocíticas, representando 3\% do total nessa espécie (KIMURA et al., 2012).

\section{MELANÓCITOS}

De acordo com Muller et al. (2001), na epiderme, podemos encontrar quatro tipos celulares distintos: Queratinócitos (85\%). Células de Langerhans (3 a 8\%); Células de Merckel (2\%) e os Melanócitos (5\%).

Os melanócitos são células dendríticas derivadas dos melanoblastos neuroectodermais e da crista neural, que durante a embriogênese migram para a epiderme, derme, membranas mucosas e olhos (SMITH et al., 2002).

$\mathrm{Na}$ camada basal da epiderme, o melanócito estabelece uma relação simbiótica com os queratinócitos. Para cada melanócito, existem cinco queratinócitos ao seu redor (HAASS et al., 2005). Ou seja, em epidermes normais, os melanócitos não estabelecem contato entre si.

Segundo Barnhill (1995), os melanócitos são células estáveis, que proliferam apenas em casos de regeneração ou injúria. Sob condições de homeostase os melanócitos permanecem em "repouso", proliferam, se diferenciam ou sofrem apoptose (HAASS et al., 2005).

A principal função dos melanócitos é sintetizar melanina, pigmento que atribui cor aos pelos e pele, protegendo esta última contra os raios solares (HSU et al., 2000).

\section{MELANINA}

De origem grega, a palavra melanina significa negro, porém, nem toda melanina apresenta coloração enegrecida. Podemos observar a eumelanina de coloração enegrecida; a feomelanina, cuja cor é amarelada ou vermelha; e a neuromelanina, que também se encontra no sistema nervoso (PERRONE, 2001). 
Segundo Muller et al. (2001), grande parte da melanina é encontrada na camada basal da epiderme, entretanto, em cães de pele escura, tal pigmento encontra-se por todas as camadas da mesma.

A síntese de melanina ocorre no interior dos melanossomas, organelas especializadas, que promovem a conversão da tirosina em melanina, no interior do Retículo Endoplasmático Granular (REG). A melanina permanece armazenada no Complexo de Golgi, e pode ser observada exclusivamente nos melanócitos.

Os melanossomas têm sua origem no aparelho de Golgi, constituem vesículas que atingem quatro estágios de desenvolvimento. Inicialmente são denominados de prémelanossomas, e à medida que sintetizam a melanina, passam a ser chamados de melanossomas (TEIXEIRA, 2011); após completada a síntese de melanina, o melanossoma recebe o nome de grânulos de melanina (BARNHILL et al., 1995).

De acordo com Camargo (2005), a melanina armazenada nos melanossomas pode ser transferida aos queratinócitos através de seus dendritos, sendo esse processo denominado citocronia.

Conforme Goldschmidt e Hendrick (2002), os melanomas podem apresentar grande quantidade de melanina (altamente pigmentados), sendo denominados de melanocíticos, ou ter falta de pigmento e são chamados de amelanocíticos.

Ainda que o grau de pigmentação não indique o potencial de malignidade dos tumores, pesquisas sugerem que melanomas amelanocíticos são mais agressivos do que os melanocíticos, pois possuem uma proliferação celular mais elevada, promovendo uma sobrevida mais curta para cães (TEIXEIRA et al., 2014).

\section{NEOPLASIAS MELANOCÍTICAS}

Segundo Camargo (2005), as neoplasias melanocíticas têm sido encontradas desde a antiguidade em indivíduos mumificados há mais de 2000 anos. Entretanto, o primeiro relato de melanoma em cães foi realizado somente em 1882, por Bunker (apud CAMARGO et al., 2008).

A transformação de melanócitos normais em neoplásicos é um processo complexo, cujo início se dá durante a mutação gênica, que gera a perda da homeostase entre o queratinócito-melanócito, seguida pela transformação celular e metástase (SMITH et al., 2002). 
De acordo com Teixeira (2011), uma vez que os melanócitos adquirem autonomia e conseguem escapar do controle dos queratinócitos, passam a se multiplicar de forma difusa, descontrolada e equivocada, levando a formação de tumores sólidos que podem assumir duas formas: benigno e maligno.

Quando a neoplasia melanocítica é benigna, recebe a denominação Malanocitoma; quando a lesão é maligna, recebe o nome de Melanoma ou Melanoma Maligno, conforme o último boletim da OMS (1998).

Conforme Head et al. (2002), os melanomas malignos podem ser subdivididos em três classes com base na sua forma celular, sendo: células redondas epitelioides e poligonais; tumor de células fusiformes (assemelha-se a fibroblastos); e tumores mistos (mostram ambos os tipos de células).

O melanoma representa um tumor localmente agressivo que irá produzir metástases para locais regionais e distantes, via linfáticos para os linfonodos regionais, ou via hematógena, para os pulmões, agravando o quadro clínico do paciente (ETTINGER e FELDMAN, 1997).

O melanoma localizado na cavidade oral é uma neoplasia altamente maligna e não é incomum que suas células tumorais migrem para outras partes do corpo ocasionando metástases em locais atípicos, tais como o cérebro, baço e coração (LIPTAK; WITHROW, 2013).

\section{ACHADOS MACROSCÓPICOS}

O melanoma, inicialmente, caracteriza-se como uma mácula preta e se transforma em uma massa firme de crescimento rápido. Pode apresentar forma de cúpula, superfície ulcerada, avermelhada e sangrante. Dependendo da quantidade de pigmento, seu interior pode ser branco-acinzentado, marrom escuro ou preto (CARLTON e MCGALVIN, 1998).

A principal característica dessa neoplasia é a manifestação de um nódulo pedunculado, solitário e bem delimitado, com variação no tamanho de 0,5 a $10 \mathrm{~cm}$ de diâmetro (SOUZA et al., 2011). 


\section{ACHADOS MICROSCÓPICOS}

Microscopicamente, o melanoma consiste de melanócitos epitelioides e fusiformes. Sendo que algumas neoplasias consistem quase que exclusivamente de células epitelioides enquanto outras são compostas de células fusiformes, semelhantes a fibroblastos. Pode-se ainda observar neoplasias de composição mista, ou seja, com presença de ambos os tipos celulares (Tabela 1).

Tabela 1. Classificação dos melanomas quanto a sua composição celular

\begin{tabular}{c|c|c}
\hline Tipo celular & Características & Localização \\
\hline $\begin{array}{c}\text { Melanomas } \\
\text { Epitelioides }\end{array}$ & $\begin{array}{c}\text { Células arredondadas; bordas discretas; } \\
\text { citoplasma abundante e vítreo; núcleo largo e } \\
\text { nucléolo proeminente. }\end{array}$ & $\begin{array}{c}\text { Região da epiderme; } \\
\text { ou membrana basal. }\end{array}$ \\
\hline $\begin{array}{c}\text { Melanomas } \\
\text { Fusiformes }\end{array}$ & $\begin{array}{c}\text { Células alongadas em forma de fuso; formam } \\
\text { ondas e feixes; estroma escasso e algumas } \\
\text { vezes em forma de ninho. }\end{array}$ & Região mesenquimal. \\
\hline $\begin{array}{c}\text { Melanomas } \\
\text { Mistos }\end{array}$ & Envolvem ambos os tipos celulares. & Ambas as regiões. \\
\hline Fonte: CARLTON e MCGALVIN (1998) (modificado)
\end{tabular}

\section{MÉTODOS DIAGNÓSTICOS}

Para diagnóstico de neoplasias orais em cães, pode-se utilizar como exames complementares: radiografias torácicas, para pesquisa de metástases pulmonares; avaliação histológica, para confirmação de suspeita clínica; radiografias e tomografias do crânio e cavidade oral, para avaliar possível comprometimento ósseo, de tecidos subjacentes e delimitar lesões (KEMPER et al., 2012).

Os linfonodos regionais (mandibular, parotídeo e retrofaríngeos mediais) devem ser examinados para observação de aumento de tamanho ou assimetria (LIPTAK; WITHROW, 2013). 
De acordo com Goldschmidt e Hendrick (2002), durante a avaliação citológica e histológica dos linfonodos, para pesquisa de metástases, deve-se atentar para a presença de melanófagos, que são comumente encontrados, uma vez que os linfonodos mandibulares realizam a drenagem do pigmento melanina da cavidade bucal para essa região, não caracterizando, portanto, indícios de metástase.

Liptak e Withrow (2013) afirmam que as preparações citológicas, por meio da aspiração por agulha fina, geralmente não são suficientes para se obter um diagnóstico definitivo, pois esses tumores são comumente infectados e inflamados, necessitando, portanto, de grande quantidade de material para análise.

A biópsia do tecido deve ser realizada para diferenciação da enfermidade benigna da maligna, e também para que os proprietários possam analisar as opções de tratamento, levando em conta o estadiamento do tumor (LIPTAK; WITHROW, 2013).

No exame histológico, podemos observar a taxa de mitose, utilizada para determinar o grau de malignidade, uma vez que melanomas altamente proliferativos, com altos índices mitóticos, que são extremamente infiltrativos aos tecidos adjacentes, representam a forma mais maligna (VAIL et al., 2007).

Visto que existem diversos graus de pigmentação melânica, o diagnóstico de melanoma pode ser considerado um desafio. Em melanomas amelanocíticos, as células tumorais não produzem melanina intracitoplasmática, por isso podem induzir a um falso diagnóstico, mimetizando histologicamente outras neoplasias como linfomas, carcinomas pouco diferenciados e tumores de células germinativas (OHSIE et al., 2008). Sendo assim, o método diagnóstico de eleição para melanomas amelanocíticos é a combinação de histopatologia com a imunoistoquímica (IHQ).

Segundo Teixeira (2011), o seguinte perfil imunoistoquímico foi preconizado para o diagnóstico de melanoma amelanocítico: Vimentina, filamento típico de células mesenquimatosas e derivados; Enolase neuroespecífica (NSE), marcador de origem neural; Proteína S-100, presente em praticamente todas as lesões de melanócitos em humanos; HMB45, anticorpo monoclonal que reage diretamente com tumores melanocíticos; Melan-A, marcador altamente sensível e específico para tumores melanocíticos.

Após o diagnóstico do melanoma, devemos estabelecer seu estadiamento clínico, o que inclui a realização de exames complementares como radiografia de tórax, 
ultrassom de abdome, tomografia computadorizada e ressonância magnética, de acordo com o critério do médico veterinário (ZYBTEK et al., 2008).

Os clínicos utilizam o termo estadiamento para determinar o quão avançada está uma neoplasia e consequentemente qual seu prognóstico. Essas categorias de estadiamento são definidas de forma diferente para cada tipo de tumor. Abaixo (Tabela 2) podemos observar a classificação dos estágios para melanoma oral em cães.

Tabela 2. Estadiamento clínico do melanoma oral

\begin{tabular}{c|c|c|c}
\hline \multirow{2}{*}{ Estágio clínico } & Tumor & Linfonodo & Metástase \\
\hline I & $<2 \mathrm{~cm}$ & - & - \\
\hline II & $2-4 \mathrm{~cm}$ & - & - \\
\hline III & $>4 \mathrm{~cm}$ ou envolvimento ósseo & Sim & - \\
\hline IV & Qualquer tamanho & Sim ou Não & Sim \\
\hline
\end{tabular}

Fonte: Organização Mundial de Saúde (1998) (modificado)

\section{PROGNÓSTICO}

De acordo com Teixeira (2011), na medicina veterinária, o prognóstico é definido somente pela análise do estadiamento clínico (TNM). Sendo que essa classificação se dá através da análise da disseminação anatômica sobre 3 aspectos: tumor primário $(\mathrm{T})$; linfonodos regionais $(\mathrm{N})$; e metástase a distância $(\mathrm{M})$.

MacEwen (2001) e colaboradores relataram tempos médios de sobrevivência para cães com melanoma oral, tratados com cirurgia, de aproximadamente 17-18, 5-6 e 3 meses com a doença de estádio I, II e III, respectivamente.

Relatos mais recentes sugerem que o melanoma oral de fase I- tratado com terapias padronizadas, incluindo cirurgia, radiação e, ou quimioterapia -tem um tempo de sobrevivência médio de aproximadamente 12-14 meses, com a maioria dos cães morrendo em decorrência de metástase distante, e não recorrência local (BERGMAN, 2010).

A classificação histopatológica de um tumor delineia o grau de malignidade, e os sistemas de classificação variam entre os tipos de tumor. Estudos demonstraram que o 
índice mitótico igual ou inferior a três indica melhor prognóstico (FONSECA et al., 2014). O grau histológico é comumente preditivo quanto à sobrevida, taxa metastática e outras variáveis clínicas.

Greene et al. (2013) sugerem que mediadores inflamatórios podem estimular ou inibir o crescimento de células tumorais, dependendo de suas concentrações. Seus trabalhos relevaram que o óxido nítrico, que é produzido por células neoplásicas e a leptina, uma substância inflamatória, estão presentes no melanoma, podendo ser dosados. $\mathrm{O}$ objetivo de seu estudo era correlacionar a presença de tais substâncias com a sobrevida dos cães. Porém, segundo análise dos autores, mais estudos devem ser realizados para determinar efetivamente esta ligação.

De uma forma geral, melanomas primários em cavidade oral de cães, possuem um prognóstico reservado (BERGMAN, 2010).

\section{TRATAMENTOS}

Os tratamentos empregados na ocorrência de melanomas incluem: excisão cirúrgica com margem de segurança de pelo menos $2 \mathrm{~cm}$; terapêuticas adjuvantes como a quimioterapia, radioterapia, imunoterapia, criocirurgia e eletroquimioterapia (QUEIROZ et al., 2004).

Para Proulx et al. (2002), em casos de melanoma em cavidade oral, a mandibulectomia parcial ou total ou a maxilectomia parcial ou total promovem uma sobrevida de até 10 meses, sem a associação de qualquer outra terapia. Porém, sem a adição de tratamento adjuvante, o risco de recidiva chega a 70\%, com média de sobrevida de 3 a 4 meses.

A Carboplatina, quando utilizada como quimioterapia de forma adjuvante, promove efeitos em cerca de 10 a $20 \%$ dos casos, sendo que apenas $2 \%$ atingem resultados satisfatórios, com uma sobrevida máxima de 9 meses (PROULX et al., 2002).

De acordo com Brockley et al. (2013), a realização de quimioterapia em associação com a ressecção cirúrgica não promoveu maior tempo de sobrevivência em seus estudos. Porém, Cancedda et al. (2014) observou que os animais que recebiam quimioterapia obtinham um tempo médio de progressão da doença maior do que o grupo que havia recebido apenas radiação. 
A radioterapia tem sido utilizada como tratamento de efeito paliativo em cães com melanoma, porém a sobrevida dos mesmos, após a terapia, é curta devido a metástases, ou recidivas locais (CUNHA et al., 2013).

Segundo Silva et al. (2006), diante da impossibilidade de ressecção cirúrgica do tumor, um tratamento alternativo seria a criocirugia, que causa danos mínimos ao tecido adjacente, é segura e pouco invasiva.

Devido ao fato de ser um tumor com características imunogênicas, a realização de imunoterapias, principalmente através de vacinas, tem sido uma estratégia bastante utilizada (GREENE et al., 2014).

O estudo realizado por Riccardo et al. (2014), para avaliar a imunogenicidade, segurança e eficácia terapêutica de uma vacina baseada em DNA humano para cães com melanoma oral, demonstrou que os cães vacinados (que adquiriram anticorpos) tiveram uma sobrevida livre da doença significativamente maior do que os cães controle não vacinados. Além da ausência de relevantes efeitos colaterais indesejáveis.

A eletroquimioterapia é a associação da eletroporação e agentes antineoplásicos, que promovem a criação de poros temporários e seletivos na membrana celular, de forma a facilitar a entrada de substâncias químicas no meio intracelular (SILVEIRA et al., 2010). Essa alternativa terapêutica permite uma maior concentração de fármacos nas células neoplásicas com baixas doses.

\section{Considerações Finais}

Apesar do prognóstico reservado, com o diagnóstico precoce, tratamento e manejo adequados, pode-se obter sobrevida maior do que a descrita em literaturas, além da melhor qualidade de vida. Podendo inclusive evitar recidivas ou metástases à distância. Entretanto, muitos estudos ainda devem ser realizados a fim de melhor compreender o comportamento de melanomas, tanto na espécie humana quanto no cão. 


\section{Referências}

BARNHILL, R. L. Pathology of melanocytic nevi and malignant melanoma. USA: Library of Congress, 1995. 294 p.

BERGMAN PJ. Cancer Immunotherapy. In: Textbook of Veterinary Internal Medicine (7th Edition), Ettinger S \& Feldman E (eds.), Elsevier Saunders Inc., St. Louis, MO, 2010, pp 2133-2137.

BROCKLEY, L.K.; et al. Malignant melanoma in 63 dogs (2001-2011): the effect of carboplatin chemotherapy on survival. New Zealand Veterinary Journal, v. 61, 2013.

CAMARGO, L. P. Neoplasias melanociticas cutâneas em cães: aspectos clínicos e histopatológicos em 58 casos. 2005. 58f. Tese (Doutorado). Universidade Federal de Viçosa, Minas Gerais, 2005.

CAMARGO, L. P.; CONCEIÇÃO, L. G.; COSTA, P. R. S.. Neoplasias melanocíticas cutêneas em cães: estudo retrospectivo de 68 casos (1996-2004). Brazilian Journal Veterinary Research Animal Science, v.45, n. 2, p.138-152, 2008.

CANCEDDA, S.; et al. Efficacy and side effects of radiation therapy in comparison with radiation therapy and temozolomide in the treatment of measurable canine malignant melanoma. Veterinary and Comparative Oncology, 2014.

CARLTON, W.W; MCGALVIN, M. O. Sistema hemopoético. Patologia veterinária especial de Thomson. 2. ed. Artmed. São Paulo. 1998. p. 23

CUNHA, S.C.S. et al. A utilização da radioterapia como terapia adjuvante no tratamento do melanoma oral em um cão. Acta Scientiae Veterinariae, 2013. Disponível em <http://www.redalyc.org/articulo.oa?id=289031818006 >. Acesso em: 13 de dezembro de 2016.

DZUTSEV, A. et al. The role of the microbiota in inflammation, carcinogenesis, and cancer therapy. Eur. J. Immunol. 2015, 45, 17-31.

ETTINGER, S. J.; FELDMAN, E. C. Tratado de Medicina interna veterinária. 4ed. São Paulo: Manole. V. 1997. p. 720-721 
FERREIRA, C. M. M.; MACEIRA, J. M. P.; COELHO, J. M. C. O. Análise imunohistopatológica, clínica e evolutiva dos melanomas. Anais Brasileiros de Dermatologia, Rio de Janeiro, v. 72, n. 2, p.: 117 - 126, 1997.

FONSECA A.K.S.; et al. Hemimaxilectomia caudal em cão com melanoma amelanocíticorelato de caso. XXIII Congresso de Pós-Graduação da UFLA, 2014. Disponível em <http://www.apg.ufla.br/resumos/resumo_2014/resumos/resumo_9_488_1.pdf >. Acesso em: 13 de dezembro de 2016.

GILLARD, M. et al. Naturally occurring melanomas in dogs as models for non-UV pathways of human melanomas. Pigment Cell Melanoma Res. 2014, 27, 90-102.

GOLDSCHMIDT M. H.; HENDRICK M. J. Tumors of the Skin and Soft Tissues. In: MEUTEN, D. J. Tumors in Domestic Animals. 4 ed. Iowa: Iowa State Press, 2002. Cap. 2, p. $78-82$.

GREENE, V.R.; et al. Expression of Leptin and iNOS in Oral Melanomas in Dogs. Journal of Veterinary Internal Medicine, v. 27, p. 1278-1282, 2013.

HAASS, N.K. et al. Melanoma progression exhibits a significant impact on connexin expression patterns in the epidermal tumor microenvironment. Histochem. Cell Biol. 2010, 133, 113-124.

HEAD, K.W.; ELSE, R.W.; DUBIELZIG, R.R. Tumors of the Alimentary Tract. In: Tumors in Domestic Animals, 4th ed.; Meuten, D.J., Ed.; Iowa State Press: Ames, IA, USA, 2002; pp. 426-430.

HSU, MEI-YU et al. Cadherin repertoire determines partner-specific gap junctional communication during melanoma progression. Journal of Cell Science. V. 113, p. 1535$1542,2000$.

KEMPER, B.; et al. Melanoma oral em cão. Relato de três casos. Medicina Veterinária (UFRPE), v.6, p. 18-23, 2012. Disponível em: <www.revista.dmv.ufrpe.br/index.php/rdmv/article/view/161>. Acesso em: 13 de dezembro de 2016.

KIMURA, K.C.; GARATE, A.P.; DAGLI, M.L.Z. Retrospective study of neoplasms in domestic animals: A survey between 1993 and 2002 of the service of animal 
pathology, department of pathology, school of veterinary medicine and animal science, University of Sao Paulo, Southeast Brazil. Braz. J. Vet. Pathol. 2012, 5, 60-69.

LIPTAK, J.M.; WITHROW, S.J. Cancer of gastrointestinal tract. In: Small Animal Clinical Oncology, 5th ed.; Eds.; Elsevier Saunders: St. Louis, MO, USA, 2013; pp. 381-395.

MACEWEEN, E. G. Tumors miscellaneos. In: WITHROW, S. J.; MACEWEEN, E. G. Small animal clinical oncology. 3. Ed. Philadephia: Saunders, 2001. Cap. 29, p. 639 -646 .

MODIANO, J.F.; RITT, M.G.; WOJCIESZYN, J. The molecular basis of canine melanoma: Pathogenesis and trends in diagnosis and therapy. J. Vet. Intern. Med. 1999, $13,163-174$.

MULLER, R. M.; SCOTT, D. W.; GRIFFIN, C. Muller and Kirk's small animal dermatology. 6. ed. Philadelphia: W.B. Saunders, 2001. 1528p.

OHSIE S.J. et al. Immuno-histochemical characteristics of melanoma. J. Cutan. Pathol. 35:433- 444. 2008.

PERRONE, E. A. Análise histological, imuno-bistoquímica e da proliferação celular de neoplasias melanociticas cutêneas caninas, estudo retrospectivo. 2001, 128f. Tese

(Doutorado). Faculdade de Medicina Veterinária e Zootecnia da Universidade de São Paulo, São Paulo, 2001.

PRIESTER,W.A. Skin tumors in domesticated animals. Data from 12 United States and Canadian Colleges of Veterinary Medicine. J. Natl. Cancer Insit. 1973, 50, 457466.

PROULX, D.R. et al. A retrospective anlaysis of 140 dogs with oral melanoma treated with external beam radiation. Radiotherapy of Oral Melanoma Dogs.v. 44, n. 3, p. 352-359, 2003.

QUEIROZ, G. F. et al. Utilização da crioterapia no tratamento de sarcoma de tecidos moles de extremidades em cães. Simpósio de Oncologia Veterinária da Universidade de São Paulo, 10. 2004, São Paulo. Resumos... São Paulo: USP, 2004. CD-ROM. 
RAMOS-VARA, J.A.. et al. Retrospective study of 338 canine oral melanomas with clinical, histologic, and immunohistochemical review of 129 cases. Vet. Pathol. 2000, 37, 597-608.

RICCARDO, F.; et al. CSPG4-specific immunity and survival prolongation in dogs with oral malignant melanoma immunized with human CSPG4 DNA. Clinical Cancer Research, v. 15, 2014.

SILVA, M.S.B.; et al. Tratamento de melanoma oral em um cão com criocirurgia. Acta Scientiae Veterinariae, v. 34, p.211-213, 2006. Disponível em: <http://www.redalyc.org/articulo.oa?id=289021868020 >. Acesso em: 13 de dezembro de 2016.

SILVEIRA, L.M.G.; et al. Utilização de eletroquimioterapia em neoplasias de origem epitelial ou mesenquimal localizadas em pele ou mucosas de cães. Brazilian Journal of Veterinary Research Animal Science, São Paulo, v. 47, p. 55-66, 2010.

SMITH, S. H.; GOLDSCHMIDT, M. H.; MACMANUS, P. M. A. A comparative review of melanocytic neoplasms. Veterinary Pathology. N.39. p. 651-678, 2002.

SOUZA, F. B. et al. Melanoma em cavidade oral de um canideo - Relato de Caso. 2011. Disponível em: <http://fio.edu.br/cic/anais/2011_x_cic/PDF/Medicinaveterinaria/MELANOMA\%20 EM\%20C AVIDADE\%20ORAL.pdf >. Acesso em: 13 de dezembro de 2016.

STARKEY, M.P. et al. Dogs really are man's best friend. Canine genomics has applications in veterinary and human medicine. Brief. Funct. Genomic. Proteomic. 2005, 4, 112-128.

TEDARDI, M.V. et al. Comparative oncology in Sao Paulo, Brazil-What to learn from cancer registry. Source J. Vet. Sci. 2014, 1, 1-8.

TEDARDI, M.V. et al. São Paulo animal cancer registry, the first in Latin America. Vet. Comp. Oncol. 2015, 13, 154-155.

TEIXEIRA, T. F. Melanomas melânicos e amelânicos da cavidade bucal de cães: aspectos epidemiológicos, morfológicos e moleculares. 2011. 139f. Tese (Doutorado). Universidade de São Paulo. Faculdade de Medicina Veterinária e Zootecnia. Departamento de Patologia, São Paulo, 2011. 
TEIXEIRA, T.F.; Cell proliferation and expression of connexins differ in melanotic and amelanotic canine oral melanomas. Veterinary Research Communications, v. 38, 2014.

VAIL, D. M.; WITHROW, S. J. Tumors of the Skin and Subcutaneous Tissues. In: WITHROW, S. J.; MACEWEN'S, E. G. Small Animal Clinical Oncology. ed. Missouri: Saunders Elsevier, 2007. Cap. 18, p. $389-393$.

ZAMBONI, R. et al. Estudo retrospectivo de melanoma canino durante o período de 2002 a 2011. In: Congresso de Iniciação Científica, Mostra Científica. 2011, Pelotas. Anais. Pelotas: UFPEL, 2011.

ZBYTEK, B. et al. Melanoma occurrence, staging and detection. Expert Review of Dermatology, v. 3, n. 5, p. 569-585, 2008. 